\title{
Exploratory Study of Relations Between Prior Knowledge, Comprehension, Disorientation and On-Line Processes in Hypertext
}

\author{
Franck Amadieu*, André Tricot and Claudette Mariné \\ University of Toulouse, CLLE-LTC, 5 Allées A. Machado 31058 Toulouse Cedex, France
}

\begin{abstract}
An exploratory experiment was carried out in order to question the relationships between prior knowledge, comprehension performance, on-line processes and disorientation in a non-linear document (hypertext). 14 participants read a non-linear document about a virus' infectiousness process which imposed to learners to construct themselves their reading orders and also a structure of the document. Correlational analyses were conducted to test the relations between the variables. Results indicated that the prior knowledge was positively correlated with the learning performance, the coherence of the reading orders, and negatively with the time spent to construct a document structure. Correlations between the prior knowledge and the disorientation indicated a marginal negative relation. The results stressed also significant correlations between the on-line processes and the comprehension performance. Interestingly, the observed correlations between the on-line processes and the disorientation suggested that the disorientation was function of difficulties to construct the document structure rather than difficulties to construct a reading order. The present study highlighted how prior knowledge constitutes resources to process non-linear documents and what type of on-line processes are related to disorientation.
\end{abstract}

\section{INTRODUCTION}

In comparison with classic linear texts, hypertexts comprehension demands cognitive processes to construct reading orders of contents and also to construct a mental representation of the contents structure. Classically, it is argued that reading hypertexts includes making decisions, understand semantic relations between information nodes, understanding spatial and/or semantic structure of hypertext [1]. Readers have to determine whether information should be found to fill in the information gaps, and to decide where he has to look for information [2]. Prior domain knowledge supplies cognitive resources to process the non-linearity for information seeking task $[3,4]$ as well as for comprehension tasks [2, 5-7]. The aim of this study was to investigate what types of cognitive processes were related to readers' prior domain knowledge and if these processes were linked to higher comprehension performance and less difficulties.

Hypertexts are non-linear documents that supply contents displayed as text sections (ex. pages, paragraphs) which are related by semantic links (ex. definitions, extensions, examples, etc.) [8]. Therefore, establishing semantic relations between text sections is a main part of hypertexts comprehension task. Processing non-linear information requires more relational processing than item specific processing [9]. Whether readers have to process semantic relations between text section to make decision and to follow a reading order, they are also involved in processing the semantic relations to construct a representation of the contents structure $[10,11]$. Although more and more studies consider coherence issues in reading orders [12-15], clues about the processes engaged in construction of a

*Address correspondence to this author at the University of Toulouse, CLLE-LTC, 5 Allées A. Machado 31058 Toulouse Cedex, France;

E-mail: amadieu@univ-tlse2.fr representation of the contents structure are less investigated. In sum, to understand the information conveyed by a hypertext, readers would have to establish semantic relations between the nodes and to construct a representation of the semantic hypertext structure. Hence, the present study proposed investigation of the on-line processes including both the selection processes (reading orders) and the structural processes of contents (construction of a document structure). The study explored the cognitive costs of these processes and their relations with comprehension performance. Besides, the study tested the role of prior knowledge on these processes, their cognitive costs and comprehension outcomes.

\section{EMPIRICAL EVIDENCES ON ON-LINE PROCESSES}

The selection of a link is a crucial cognitive task because the comprehension may be affected by the level of coherence between the initial text section and the target text section $[14,16]$. Linear and hierarchical structures help readers to select coherent links leading them to follow to systematic reading orders of the structure [14, 17-19].

Investigations on navigation stressed that high prior knowledge users conduct explorations more detailed and more in depth than low prior knowledge users [20,21]. High prior knowledge helps learners to follow more structured navigational patterns [22] and less sequential exploration [23]. One explanation is that high prior knowledge learners would be able to identify semantic relations between text sections and, hence, to construct more coherent reading orders [12].

Other empirical works showed that coherence of reading orders impact comprehension performance according to the level of prior knowledge. Whereas following high coherent reading orders lead low prior knowledge readers to high comprehension scores, following low coherent reading 
orders lead high prior knowledge to high comprehension scores $[14,15]$. Prior knowledge supplies resources to conduct inferences in orders to fill in coherence gaps between text sections (i.e. nodes). That is corroborated by longer time allocated to process low coherent text for high prior knowledge readers [24]. Processing low coherence between text sections would be cognitively costly for low prior knowledge readers [13].

Several findings suggest that another main cognitive requirement concerns the construction of a mental representation of the contents macro-structure. [25] as well as [26] showed that prior knowledge supported comprehension only for the macro-level of texts representations. These results suggest that prior knowledge would support construction of an overall organization of the contents. [27] highlighted that high prior knowledge learners paid less attention than low prior knowledge learners to macro-concepts of a hierarchical non-linear document. This finding is consistent with facilitated processing of hypertext structures by prior knowledge. Nerveless, further investigations on these effects are needed.

\section{EMPIRICAL EVIDENCES ON DISORIENTATION}

The cognitive requirements exposed in the previous section suggest that navigating in a non-linear document may impose cognitive processes that mobilize resources in working memory and may entail high cognitive load. Hence, readers' disorientation may reflect difficulties to cope with the requirements of the task. Many authors argue that when the task requirements are high, readers would experience disorientation problems [3, 28-31]. For instance, a high number of embedded links and a large semantic distance would have a negative impact on cognitive load and disorientation [32]. However empirical evidences are still needed to support this claim.

According to Cognitive Load Theory, disorientation could reflect an extraneous cognitive load in learning from hypertexts because it is the consequence of ineffective processes in learning [12,27]. Extraneous cognitive load is a form of cognitive load that should be avoided to reach efficacy material for learning [33]. In the theoretical framework of Cognitive Load Theory, cognitive load depends on the interaction between task features and learners' features [34] and can be measured by assessment of mental effort and learning performance [35].

In hypertexts, high prior knowledge learners seem to be less disoriented than low prior knowledge learners [3, 21, 22]. Nevertheless, other studies did not observe any effect of prior knowledge on subjective disorientation [12, 36-39]. Furthermore, difficulty to prove relations between disorientation and prior knowledge is illustrated by [40]. The results did not indicate any relations between disorientation and prior knowledge, whereas a negative correlation between learning performance and disorientation and a positive correlation between learning performance and prior knowledge were observed. Too few studies have investigated the effects of prior knowledge on hypertext comprehension including disorientation measures. That is why, in the present study three dimensions were examined in relation with prior domain knowledge: comprehension performance, online processes and disorientation.

\section{EMPIRICAL EVIDENCES ON COMPREHENSION PERFORMANCE}

Lack of guidance in hypertexts imposes high cognitive requirements, whereas guidance as overviews or hierarchical structures may limit these requirements and promote better comprehension performance for low prior knowledge readers (e.g. [10]). Prior knowledge helps to reach higher comprehension performance in case of hypertext without guidance [36, 41-43].

Considering different levels of representation, [18] showed positive impact of prior knowledge only on situation model. Conversely, studies $[14,25]$ obtained positive effect of prior knowledge only on the text based representation. However, others studies showed positive effects on both levels of representation [38, 39]. Recently, studies highlighted positive effect of prior knowledge on construction of macrostructure of contents representations [25-26]. Overall, the inconsistencies in the literature on the type of representation (text based representation, situation model) influenced by prior knowledge prompt calls for further clarification of prior knowledge effects.

\section{PURPOSES AND HYPOTHESES}

In the present study there were two main purposes. Firstly, understanding the relations between prior knowledge and three dimensions implied in hypertexts comprehension, that is, contents representations, navigation and cognitive load. The contents representations concerned text base and situation model [44]. Navigation processes were reflected by the coherence of reading orders and the behaviors for construction of a document structure during the comprehension task. Global cognitive load was reflected by mental effort ratings whereas extraneous cognitive load was reflected by conceptual disorientation ratings. Therefore, the disorientation measure was expected to be a more sensitive measure of cognitive load than the mental effort measure. Secondly, the present study tended to understand also the relations between all these dimensions. Testing these relations should provide information about hypertexts comprehension and the requirement processes of the task.

As regard to the first main purpose, domain's deep principles of experts [45-47] are crucial to process new situations. Therefore, in the present study, effects of prior knowledge were considered as effects of general domain principles. Prior knowledge was expected to support comprehension of a non-linear document and active explorations of the document based on the domain principles. That is to say, it was hypothesized high levels of prior knowledge would be related to high scores of deep comprehension (requiring numerous inferences: situation model), construction of high coherent reading orders, better construction of hypertext structure and a low cognitive load.

As regard to the second purpose, it was hypothesized, on the one hand, that high comprehension performance will be related to high coherent reading orders and a more efficient construction of the document structure, and on the other hand, that high comprehension performance will be also related to low cognitive load. Besides, it was hypothesized that low coherent reading orders and a long time to construct 
the document structure would reflect difficulties, and thus, would be linked to high disorientation.

\section{METHOD}

\section{Participants}

In order to study high level of prior knowledge in the studied domain (i.e. virology), Master grade students in biology were recruited. Fourteen Master grade students (6 females and 8 males) in biology and health took part in the experiment. During the Master, all participants had attended courses about the different types of viruses and their multiplication cycles. The mean age of the participants was 23.79 years (from 22 to 26 years).

\section{Material and Measures}

\section{Learning Material}

A non-linear document in domain of virology was designed for the experiment. The course dealt with the multiplication cycle of a virus (cycle of coronavirus). Coronavirus was chosen because it was not familiar to the participants and thus it was supposed to allow important knowledge acquisition. The content was made up of different concepts (elements, events/actions). The main concepts have been isolated in different text sections (i.e. information nodes).

The concept map displayed 13 concepts without any links between the concepts ( 3 concepts about the virus' characteristics and 10 about the events and actions of the multiplication cycle). At the start, the structure of the map was randomly organized on screen and identical for all participants. To read the text sections, the participants clicked on the corresponding concepts and they were allowed to move the concepts using the mouse to structure the map from the start of the learning phase. Each text section was understandable alone in order to participants focused their comprehension processes on relations between concepts rather than on individual concepts.

Using the non-linear document implied two level of task: selecting text section (reading order) and constructing a concepts-map organization. To read text sections concepts, participants clicked on a link of the concept map to open a text section and then on a link to come back to the map and open a new concept (or the same concept). Organizing the concept map consisted of changing positions of the concepts. That is, the displayed concepts were movable thanks to mouse actions (drag \& drop). This dimension of the using task was expected to require relational and structural processing and to impose additional processing. The starting display of the concepts reflected a random organization and was exactly the same for all participants (see Fig. 1).

The construction task of the document structure was expected to promote higher engagement in the relational processes of contents and also offered a tool to assess these processes. Indeed, this type of task is considered as requiring relational processes between the map-concepts and thus encourages mobilization and construction of structural knowledge [48].

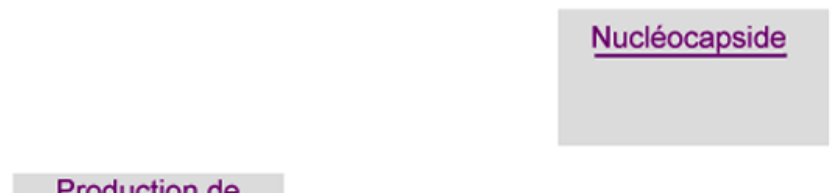

\section{Production des} génomes viraux

\section{protéines virales}

\section{Production d'ARN} messager
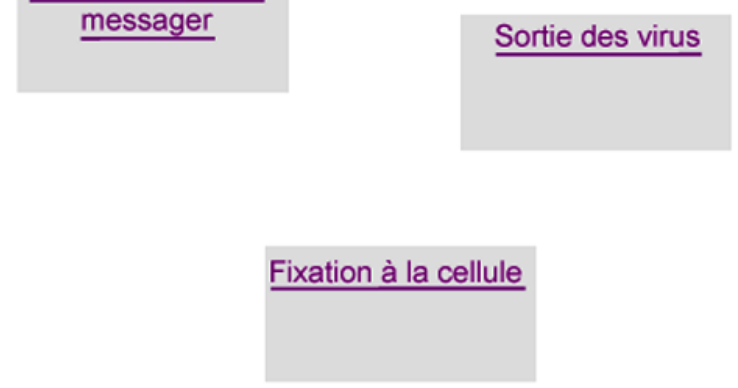

Fusion de la membrane

\section{Génome viral}

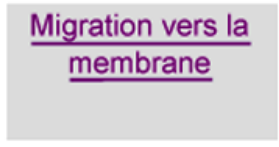

Polymérase

Migration des protéines virales

Fig. (1). Starting configuration of the concepts displayed on screen. 


\section{Coherence of Reading Orders}

The activity of the participants was recorded with the freeware "Traceur Internet 0.02.0027" (from the French national institute for research on pedagogy). Activations of each link (opened concept) and time spent reading each text section and concept map were recorded.

Similarly to a previous study [12], the assessed coherence of reading orders was temporal-causal (i.e. navigating respecting the temporal and causal relations between elements and events of the virus' multiplication cycle exposed in the different text sections). The level of coherence of the reading orders was calculated measuring the distance between the participants' reading orders and the chronological sequence of the multiplication cycle of the virus. For instance, when a participant jumped from node A to node $\mathrm{C}$ or from node $\mathrm{C}$ to node $\mathrm{A}$, the distance was recorded as "2". A jump respecting exactly the chronology (e.g. from node $\mathrm{D}$ to node $\mathrm{E}$ or node $\mathrm{E}$ to $\mathrm{D}$ ) received a distance score of " 1 ". The coherence score of the reading orders equals the mean distance score. Therefore the more the coherence score tended to " 1 ", the more the reading order was coherent.

\section{Measure of Difficulties for Constructing the Concept-Map Structure}

The manipulation of the map concepts was recorded thanks to "Camtasia Studio v. 3.0". The software recorded all the events on the screen and produced a movie file (avi file). Therefore, the time taken to organize the concepts on screen was analyzed in order to assess the difficulties of the participants to construct a document structure. That is to say, the time taken until the concepts reach a final organization was considered (i.e. the participant stopped moving the concepts). This time reflected problems to construct a satisfying structure and the need to consult the text sections (i.e. extract information) to organize afterwards the concepts.

\section{Measure of Prior Domain Knowledge}

To assess level of prior knowledge in the domain of the virus cycles (domain principles), the participants were asked to write on a sheet of paper the principles and particularities of the viruses' multiplication cycles. They were instructed to indicate main stages and sub-stages of the different multiplication cycles of viruses and to enumerate the different particularities they knew (i.e. the differences between the viruses and their cycles). Based on virology books dealing with the viruses' multiplication cycles, each stage and element was scored by one point (max: 49). The mean prior knowledge score of participants was $17.36(S D=$ 4.13) with a range from 11 to 26.

\section{Comprehension Performance}

A statement judging task was used to assess two types of content representations: text base and situation model. Answering the text based items required the participants to retrieve from memory information explicitly mentioned in a text section in one or two sentences. Answering the situation model items required participants to establish conceptual links between two or more text sections (the participants had to possess a representation of implicit relations between concepts).
Eleven statements assessed text based representation and 13 statements assessed situation model. Participants were presented with 24 statements about the coronavirus cycle. The statements were displayed on a screen by the software Inquisit 2.0.51002 (Millisecond Software LLC, 2005). The participants were instructed to judge the statements by indicating "right", "wrong", or "I do not know" (included to avoid random answers) by pressing keys $\mathrm{P}$ (upper right corner), A (upper left corner), or spacebar (low middle), respectively. Each correct judgment scored one point.

\section{Mental Effort and Disorientation Measures}

Mental effort corresponds to the cognitive capacity allocated to cope with the task demand. Hence mental effort would reflect a global cognitive load (i.e. encompassing the three forms of cognitive load). The invested mental effort to learn the lesson was measured using the subjective 9-points rating scale designed by Paas [35] ("Please indicate how much mental effort you invested in studying the learning task": 1 = "very very low", 9 = "very very high").

Perceived disorientation was measured using a part of the set of subjective rating scales [49]. Five 9-points rating scales ( 1 = "very, very low", $9=$ "very, very high") were selected to assess conceptual disorientation (conceptual disorientation refers to the users' difficulties to link the different concepts conveyed by a hypertext [50]. Indeed, we were interested in the construction of meaning from a nonlinear document and not in the construction of a physical representation of the document. The scales were modified according to our material and they assessed the perceived difficulty. The scales corresponded to "your difficulty for": (a) "understanding the relationships between the different pages of the document was", (b) "knowing which page to consult next was", (c) "knowing your location in the lesson was", (d) "finding information that you have already read was", and (e) "understanding the sequence of the virus' multiplication cycle was". The five disorientation scales showed a strong internal reliability (Cronbach's Alpha = 0.86). A mean disorientation score was computed from scores of the five rating scales.

\section{Procedure}

The 14 participants were tested in group. The session duration was approximately 50 minutes. 24 hours before the main session, all participants answered the prior knowledge test. Each participant received a prior knowledge score.

To limit use difficulties of the document functions, each participant performed a familiarization task during five minutes in another domain (restaurant menus). Participants started the comprehension phase when they considered they know well how to use functions.

For the comprehension task, they were instructed to study the documents in order to understand and learn how the virus infects a cell. The name and the type of the virus were not mentioned in the instruction nor in the document to promote use of prior domain knowledge of participants. They had 15 minutes to study the non-linear document.

After the comprehension phase, the participants rated the mental effort to learn the lesson and the disorientation that they experienced. Next, the participants were asked to report the strategies they follow to consult the document. Then they 
Table 1. Pearson's Correlations Between the Prior Knowledge Scores, the Learning Outcomes Scores, the Mental Effort Ratings, the Disorientation Ratings, the Coherence Scores of the Reading Orders and the Time to Reach the Final Organization of the Map

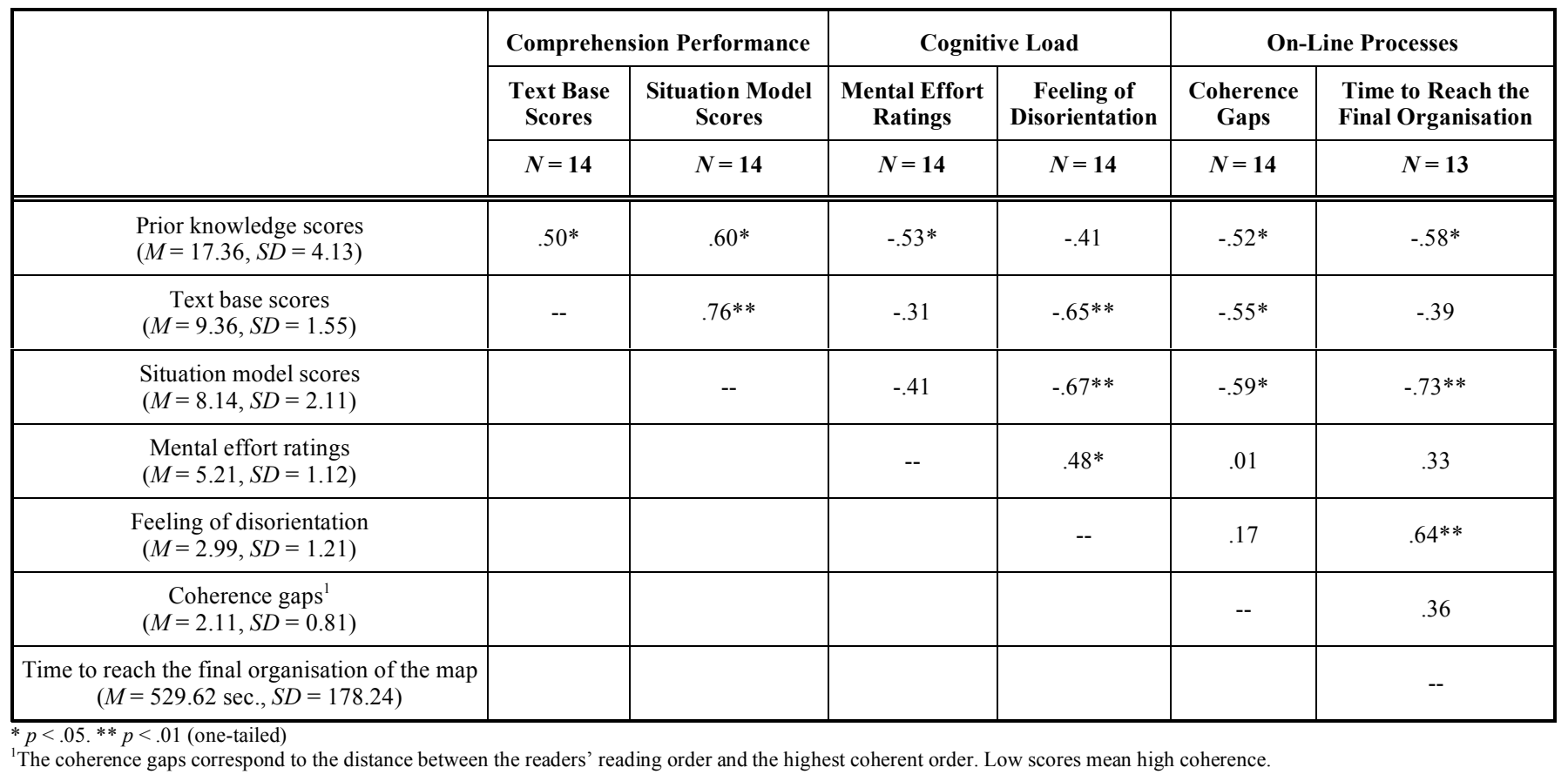

performed the judgment tasks. The set of statements linked to explicit information were introduced before the set of statements linked to implicit information because the second set might help participants to judge the first set.

\section{RESULTS}

Because the sample included only 14 participants, all the analyses consisted in calculating Pearson's correlations (oneway) between the different measures. The normality assumption was satisfied for each variable. The correlation coefficients are presented in Table $\mathbf{1}$.

\section{Correlations Between Prior Knowledge Scores and Others Variables}

Unexpectedly, a positive correlation was also observed between the prior knowledge and the text base scores. However, in accordance with our expectations, the analyses revealed that the prior knowledge scores were positively correlated with the situation model scores.

The correlation with the mental effort rating confirmed also our assumptions. The prior knowledge was negatively correlated with the mental effort ratings. Nevertheless the negative correlation between the prior knowledge scores and the disorientation ratings was only marginally significant ( $p$ $=.07)$.

In order to control the reading times, correlations between the prior knowledge and the global reading time, reading time of the map, reading time of the text sections and the number of consultations of the text sections were computed. The analyses did not indicate any relations.

For the construction of reading orders, it was hypothesized that a high level of prior knowledge would be linked to more coherent reading orders. All participants reported that they tried to follow the temporal-causal sequence to construct their reading orders. As expected, the analyses computed between the prior knowledge scores and the coherence of the reading orders indicated that the coherence of the reading orders increased with the prior knowledge. This result corroborated that the effective use of reading strategies based on the chronology of the infectiousness process increased with prior knowledge.

For the construction of the document structure, it was expected that participants with high prior knowledge would organize the concepts of the map on the basis of their knowledge structures (i.e. domain principles). Hence high prior knowledge should be linked to a faster organization of the concepts map and to a final organization similar to the sequence of the virus' infectiousness process. One participant was removed from the analysis conducted on the time to construct a document's structure: he or she initialized the spatial organization during the learning task. Qualitatively, 11 participants out of 14 produced an organization of the concepts relying on the chronology of the multiplication cycle (an example of final organization is given in Fig. (2)). However, although most of participants produced similar structures, the duration to reach a final organization of the map correlated negatively with the prior knowledge scores. In other words, the efficiency to construct the document structure was positively related to the prior knowledge.

Correlations Between Mental Effort and Disorientation Ratings, Comprehension Performance Scores and OnLine Measures

In spite of a significant positive correlation between the mental effort rating and the disorientation, only the disorientation ratings were significantly correlated with all 


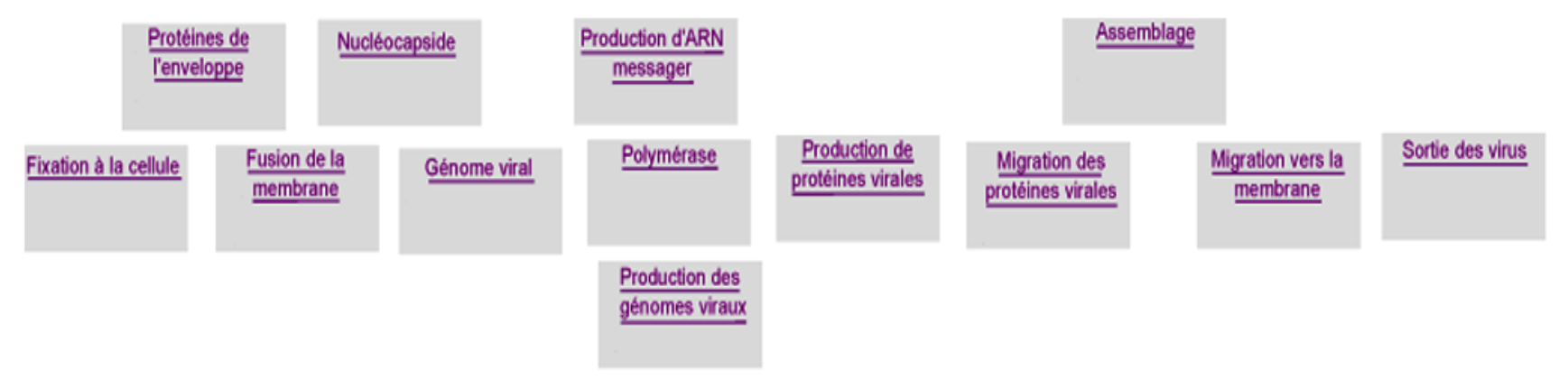

Fig. (2). Example of a final organization of concepts produced by one participant.

performance measures. High disorientation was linked to low performance. That is consistent with our expectation of a performance hampered by high extraneous cognitive load.

In accordance with our hypotheses, the coherence of the reading orders and the time to reach the final organization of the concepts were globally correlated with the performance measures. That is, low coherence of the reading orders and long time taken to organize the concepts were related to low performance.

However, contrary to our expectations, both the mental effort and disorientation ratings did not correlate with the coherence of the reading orders. Nevertheless the time taken to reach the final configuration of the map concepts was positively related to the disorientation. This result suggests that disorientation would depend on difficulties to structure the contents rather than difficulties to make decisions on the reading orders.

\section{DISCUSSION}

The present study investigated the processes engaged in hypertexts comprehension. The relations between prior knowledge, comprehension performance, on-line processes and perceived disorientation were explored. An original task consisting of construction of a non-linear document structure during comprehension task was designed. This task was expected to promote relational and structural processes and to efficiency of these processes. The classic experiments recorded only reading orders and did not have on-line clues about how readers construct a mental representation of the hypertext structure.

\section{Comprehension Performance, On-Line Processes and Disorientation}

The correlation analyses between performance, on-line processes and disorientation provided clues about the nature of such processes. As expected, high performance was linked to high coherent reading orders, short time to construct concepts structure and low disorientation. Therefore, these results suggest that establishing semantic relations between two nodes (text section) and structuring the text sections are part of hypertexts comprehension. These cognitive processes are required to reach high comprehension performance.

Besides, an interesting result was obtained on the relations between the recorded on-line processes and the disorientation ratings. Whereas the perceived disorientation and the coherence of the reading orders were unrelated, high disorientation was related to great time taken to reach the final organization of the concepts of the map. This result emphasizes that disorientation would be more dependent on processing engaged in constructing a representation of an overall organization of information than on processing engaged in constructing only relations between two nodes. Further research should explore in more depth the types of processing causing disorientation (e.g. how learners construct a meaningful representation of hypertext 
structures) and how demanding these types of processing are. Because the designed task for the present study led readers to structural and relational processes, the extent of these processes should be also tested using other materials and tasks.

\section{Prior Knowledge and Hypertexts Comprehension}

A positive relation between prior knowledge and coherence of the reading orders was obtained. Thus, the investigations of the reading orders provided some evidence for the existence of active explorations based on prior domain knowledge. This result corroborates previous findings showing that prior knowledge lead readers to follow more coherent reading orders in non-linear documents [12]. However, that is not consistent with the works conducted by Salmerón and his colleagues $[14,15]$ which showed that high prior knowledge readers benefited from low coherent reading orders. Nevertheless, we argue that those different results are compatible. Indeed, contrary to our experiment, the Salmerón's experiments included non-linear document highly structured (i.e. hierarchy or matrix), the participants followed systematic reading orders according to the structure. In the present study, the participants were not guided by a given structure of the document; hence there were forced to establish coherence to construct their reading orders.

The analyses conducted on the on-line processes to construct the structure of the concepts map provided information to help in understanding how readers process hypertext structure. Most of the participants constructed similar document structures (based on the domain principles). The most interesting result concerns the time to construct the document structure. The correlation analyses indicated that if learners had high prior knowledge, time taken to organize the document structure was shorter. This finding suggests that readers would produce inferences based on their knowledge structures to find relations between text sections (nodes) and construct a semantic structure of the whole document with more efficiency.

Considering the relation between prior knowledge and disorientation, the more the learners had prior knowledge, the less they expressed mental effort and disorientation feelings, although this later correlation did not reach significance. The correlation results of the study corroborated the classical positive relation between prior knowledge and comprehension performance. However a positive correlation between prior knowledge and text base scores was not consistent with our hypotheses which predicted effects only on situation model scores. It may suggest that readers' prior knowledge would be useful to construct deep representation as well as shallow representation of the contents. This finding is consistent with previous findings showing effects on learning of explicit and implicit information [26, 38].

Overall, the results support our hypotheses; prior knowledge is linked to higher comprehension performance, more coherent reading orders, more efficient processes to construct the document structure and lower experienced cognitive load.

\section{Future Research}

\section{Development of Cognitive Load Measurements in Hypertexts Comprehension}

The present findings indicated that measuring cognitive load using different measure may produce interesting data. The experiment showed a significant relation, but moderate, between mental effort and disorientation. This result is consistent with our claim that disorientation would be view as a part of a more general construct of cognitive load, measuring extraneous cognitive load [27]. Disorientation and global cognitive load would be distinct constructs. The analyses showed that disorientation appeared more related to others variables than mental effort (i.e. global cognitive load). It suggests that disorientation is a relevant construct to study extraneous cognitive load in comprehension of nonlinear documents, but it still requires accurate developments. Besides, using objective measures of disorientation should give more reliability. Most of the experiments use subjective measures and suffer methodological limits of such subjective measures [51]. In the research area on information search tasks with hypertexts, objectives measures based on navigation behaviors were developed [52, 53]. Future experiments should include such objective measures in addition to subjective measures.

\section{Investigating the Effects of Knowledge Structures}

The present study showed that knowledge structures may explain how readers select information and structure hypertext contents. These relational and structural processes depended on the principals of the knowledge domain. Therefore, studying prior knowledge should imply investigations of knowledge structures rather than considering prior knowledge through a quantitative approach. For instance, experimental works stressed that a previous mental representation of contents may guide subsequent processing of the same contents: a previous mental representation structured in a similar way with a document structure leads readers to higher text base scores [54] or to better processing of details [27], but not to higher situation model scores. Different techniques to measure knowledge structure are available: for example, the technique of pathfinder networks [55] or the technique of cards clustering [56].

\section{On-line Processes Engaged in Construction of Contents Structure}

Further investigations on the nature of processes engaged in hypertexts comprehension and their interrelations are needed. Continuing isolation of different types of processes should help us to improve our understanding of navigation and comprehension with hypertexts. However, a process may depend on another process. For instance, in the case of the present study, we may hypothesize that relational process for establishing semantic relations between the text sections was part of structural processes for constructing the document structure. Studying how such processes evolve during task should inform on relations between the types of processes. 


\section{ACKNOWLEDGEMENT}

The present research was not funded by any organization or institution.

\section{REFERENCES}

[1] Dillon A, Vaughan M. "It's the journey and the destination": shape and the emergent property of genre in evaluating digital documents. New Rev Multimed Hypermed 1997; 3: 91-106.

[2] Shapiro A, Niederhauser D. Learning from hypertext: research issues and findings. In: Jonassen DH, Ed. Handbook of research on educational communications and technology. Mahwah: Lawrence Erlbaum Associates 2004; pp. 605-20.

[3] McDonald S, Stevenson RJ. Effects of text structure and prior knowledge of the learner on navigation in hypertext. Hum Factors 1998; 40(1): 18-27.

[4] Rouet JF. What was I looking for? The influence of task specificity and prior knowledge on students' search strategies in hypertext. Interact Comput 2003; 15(3): 409-28.

[5] Moos DC, Azevedo R. Self-regulated learning with hypermedia : The role of prior domain knowledge. Contemp Educ Psychol 2008; 33(2): 270-98.

[6] Scheiter K, Gerjets P. Learner control in hypermedia environments. Educ Psychol Rev 2007; 19(3): 285-307.

[7] Shapiro A. Hypermedia design as learner scaffolding. Educ Tech Res Dev 2008; 56(1): 29-44.

[8] Rouet JF, Levonen JJ, Dillon A, Spiro RJ. An introduction to hypertext and cognition. In: Rouet JF, Levonen JJ, Dillon A, Spiro RJ, Eds. Hypertext and cognition. Mahwah: Lawrence Erlbaum Associates 1996; pp. 3-8.

[9] Wenger MJ, Payne DG. Comprehension and retention of nonlinear text: Considerations of working memory and material-appropriate processing. Am J Psychol 1996; 109(1): 93-130.

[10] de Jong T, van der Hulst A. The effects of graphical overviews on knowledge acquisition in hypertext. J Comput Assist Learn 2002; 18(2): 219-31.

[11] Farris JS, Jones KS, Elgin PD. Users' schemata of hypermedia: What is so spatial about a website? Interact Comput 2002; 14: 487502 .

[12] Amadieu F, Tricot A, Mariné C. Prior knowledge in learning from a non-linear electronic document: Disorientation and coherence of the reading sequences. Comput Hum Behav 2009; 25(2): 381-8.

[13] Madrid RI, van Oostendorp H, Melguizo MCP. The effects of the number of links and navigation support on cognitive load and learning with hypertext: the mediating role of reading order. Comput Hum Behav 2009; 25: 66-75

[14] Salmerón L, Cañas JJ, Kintsch W, Fajardo I. Reading strategies and hypertext comprehension. Discourse Process 2005; 40(3): 171-91.

[15] Salmerón L, Kintsch W, Cañas JJ. Reading strategies and prior knowledge in learning from hypertext. Mem Cognit 2006; 34 (5): 1157-71.

[16] Storrer A. Coherence in text and hypertext. Doc Des 2002; 3(2): $156-68$.

[17] Foltz PW. Comprehension, coherence and strategies in hypertext and linear text. In: Rouet JF, Levonen JJ, Dillon A, Spiro RJ, Eds. Hypertext and Cognition. Mahwah: Lawrence Erlbaum Associates 1996; pp. 109-36.

[18] Shapiro AM. The relationship between prior knowledge and interactive overviews during hypermedia-aided learning. J Educ Comput Res 1999; 20(2): 143-67.

[19] Zeller P, Dillenbourg P. Effet du type d'activité sur les stratégies d'exploration d'un hyperdocument. Sci Tech Éduc 1997; 4(4): 41335 .

[20] Carmel E, Crawford S, Chen H. Browsing in hypertext: a cognitive study. IEEE Trans Syst Man Cyber 1992; 22(5): 865-84.

[21] Jenkins C, Corritore CL, Wiedenbeck S. Patterns of information seeking on the Web: a qualitative study of domain expertise and Web expertise. IT Soc 2003; 1(3): 64-89.

[22] Mishra P, Yadav A. Using hypermedia for learning complex concepts in chemistry: A qualitative study on the relationship between prior knowledge, beliefs, and motivation. Educ Info Tech 2006; 11(1): 33-69.

[23] MacGregor SK. Hypermedia navigation profiles: cognitive characteristics and information processing strategies. J Educ Comput Res 1999; 20(2): 189-206.
[24] Salmerón L, Baccino T, Cañas J. How prior knowledge and text coherence affect eye fixations in hypertext overviews. In: Sun R, Miyake N, Eds. In: Proceedings of the $28^{\text {th }}$ Annual Conference of the Cognitive Science Society. Hillsdale: Lawrence Erlbaum Associates 2006; pp. 715-9.

[25] Le Bigot L, Rouet JF. The impact of presentation format, task assignment, and prior knowledge on students' comprehension of multiple online documents. J Lit Res 2007; 39(4): 445-70.

[26] Potelle H, Rouet JF. Effects of content representation and readers prior knowledge on the comprehension of hypertext. Int J Hum Comput St 2003; 58(3): 327-45.

[27] Amadieu F, van Gog T, Paas F, Tricot A, Mariné C. Effects of prior knowledge and concept-map structure on disorientation, cognitive load, and learning. Learn Instr 2009; 19: 376-86.

[28] Conklin J. Hypertext: an introduction and survey. IEEE Comput 1987; 20(9): 17-41

[29] Mohageg MF. The influence of hypertext linking structures on the efficiency of information retrieval. Hum Factors 1992; 34(3): 35167.

[30] Niederhauser DS, Reynolds RE, Salmen, DJ, Skolmoski P. The influence of cognitive load on learning from hypertext. J Educ Comput Res 2000; 23(3): 237-55.

[31] Wright P. Cognitive Overheads and prostheses: some issues in evaluating hypertexts. In: Furuta R, Stotts D, Eds. In: Proceedings of the Third ACM Conference on Hypertext. New York: ACM Press 1991; pp. 1-12.

[32] DeStefano D. LeFevre JA. Cognitive load in hypertext reading: a review. Comput Hum Behav 2007; 23: 1616-41

[33] Sweller J, van Merrienboer J, Paas F. Cognitive architecture and instructional design. Educ Psychol Rev 1998; 10(3): 251-96.

[34] Paas F, van Merriënboer J. Instructional control of cognitive load in the training of complex cognitive tasks. Educ Psychol Rev 1994; 6(4): 351-71

[35] Paas, F. Training strategies for attaining transfer of problemsolving skill in statistics: a cognitive-load approach. J Educ Psychol 1992; 84: 429-34.

[36] Calisir F, Eryazici M, Lehto MR. The effects of text structure and prior knowledge of the learner on computer-based learning. Comput Hum Behav 2008; 24(2): 439-50.

[37] Calisir F, Gurel Z. Influence of text structure and prior knowledge of the learner on reading comprehension, browsing and perceived control. Comput Hum Behav 2003; 19(2): 135-45.

[38] Müller-Kalthoff T, Möller J. The effects of graphical overviews, prior knowledge, and self-concept on hypertext disorientation and learning achievement. J Educ Multimed Hypermed 2003; 12(2): 117-34.

[39] Müller-Kalthoff T, Möller J. Browsing while reading: effects of instructional design and learners' prior knowledge. ALT-J 2006; 14(2): 183-98.

[40] Baylor AL. Perceived disorientation and incidental learning in a web-based environment: internal and external factors. J Educ Multimed Hypermed 2001; 10(3): 227-51.

[41] Lee SS, Lee YHK. Effects of learner-control versus programcontrol strategies on computer-aided learning of chemistry problems: For acquisition or review? J Educ Psychol 1991; 83(4): 491-8.

[42] Recker M, Pirolli P. Modeling individual differences in student's learning strategies. J Learn Sci 1995; 4(1): 1-38.

[43] Shin E, Schallert D, Savenye C. Effects of learner control, advisement, and prior knowledge on young students' learning in a hypertext environment. Educ Tech Res Dev 1994; 42(1): 33-46.

[44] Kintsch W. The role of knowledge in discourse comprehension: A construction-integration model. Psychol Rev 1988; 95(2): 163-82.

[45] Briggs P. They know what they're doing? An evaluation of wordprocessor users' implicit and explicit task-relevant knowledge, and its role in self-directed learning. Int J Man-Mach Stud 1990; 32(4): 385-98.

[46] Chi MTH, Feltovich PJ, Glaser R. Categorization and representation of physics problems by experts and novices. Cogn Sci 1981; 5(2): 121-52.

[47] Dee-Lucas D, Larkin JH. Novice rules for assessing importance in scientific texts. J Mem Lang 1988; 27(3): 288-308.

[48] Jonassen DH. Effects of semantically structured hypertext knowledge bases on users knowledge structures. In: McKnight C, Dillon A, Richardson J, Eds. Hypertext: a psychological perspective. New York: Ellis Horwood 1993; pp. 153-68. 
[49] Ahuja J, Webster J. Perceived disorientation: An examination of a new measure to assess web design effectiveness. Interact Comput 2001; 14(1): 15-29.

[50] Cress U, Knabel OB. Previews in hypertexts: effects on navigation and knowledge acquisition. J Comput Assist Learn 2003; 19(4): 517-27.

[51] Cegarra J, Chevalier A. The use of Tholos software for combining measures of mental workload: towards theoretical and methodological improvements. Behav Res Methods Instrum Comput 2008; 40(4): 988-1000.
[52] Gwizdka J, Spence I. Implicit measures of lostness and success in web navigation. Interact Comput 2007; 19(3): 357-69.

[53] Otter M, Johnson H. Lost in hyperspace: metrics and mental models. Interact Comput 2000; 13(1): 1-40.

[54] Mannes SM, Kintsch W. Knowledge organization and text organization. Cognit Instruct 1987; 4(2): 91-115.

[55] Goldsmith TE, Johnson PJ, Acton WH. Assessing structural knowledge. J Educ Psychol 1991; 83(1): 88-96.

[56] Hsu YC. The effects of metaphors on novice and expert learners performance and mental-model development. Interact Comput 2006; 18(4): 770-92.

(C) Amadieu et al.; Licensee Bentham Open.

This is an open access article licensed under the terms of the Creative Commons Attribution Non-Commercial License (http: //creativecommons.org/licenses/by$\mathrm{nc} / 3.0 /$ ) which permits unrestricted, non-commercial use, distribution and reproduction in any medium, provided the work is properly cited. 